\title{
Effect of Oxygen Concentration on the Detection of Mercury in an Atmospheric Microwave Discharge ${ }^{1}$

\author{
K. Hadidi², P.P. Woskov, G.J. Flores, K. Green, P. Thomas \\ Plasma Science and Fusion Center, Massachusetts Institute of Technology \\ Cambridge, MA 02139, USA
}

\begin{abstract}
A microwave plasma combined with atomic emission spectroscopy is being developed for real time emission monitoring of metals in smokestacks. The plasma is sustained by 1.5 $\mathrm{kW}, 2.45 \mathrm{GHz}$ microwave radiation in a gas flow at atmospheric pressure. The addition of oxygen to a nitrogen gas stream reduces dramatilcally the emission intensity of the 253.65 $\mathrm{nm}$ mercury transition, while at the same time it increases the emission intensity of the 405.78 lead line and other metal transitions. Measurements as a function of oxygen concentration in the plasma gas stream on the intensity of the $253.65 \mathrm{~nm}$ mercury line and on the excitation temperature, $\mathrm{T}_{\mathrm{exc}}$, are presented. Possible mechanisms in the plasma that can effect the emission strength of the $\mathrm{Hg}$ atom are explored. Plasma absorption at $253.65 \mathrm{~nm}$ by ozone is ruled out by UV transmission measurements. The measurements suggest that a slight cooling of $\mathrm{T}_{\mathrm{exc}}$ with the addition of oxygen, combined with the sharp electron impact excitation cross section can explain the observed behavior.
\end{abstract}

KEYWORDS: Emission monitoring of metals, mercury detection, smoke stacks, atmospheric microwave discharge, excitation temperature

1 Supported by the Office of Science and Technology, U.S. DOE.

${ }^{2}$ Corresponding author: Hadidi@psfc.mit.edu 


\section{INTRODUCTION}

Throughout the world, legislation is being tightened to reduce the emission of hazardous metals such as mercury and arsenic from power plants, thermal processing plants, and other industrial sources. In the USA, the Environmental Protection Agency (EPA) is planning to tighten emission regulations of at least 7 of the most hazardous metals $(\mathrm{Hg}$, $\mathrm{As}, \mathrm{Sb}, \mathrm{Pb}, \mathrm{Be}, \mathrm{Cr}$, and $\mathrm{Cd}$ ). However, to enforce the new proposed laws, measurement instrumentation must be made available to these industries to insure compliance with the new emission limits. At the present time, there is only one commercial real time metals monitoring system available on the market ${ }^{1}$. Improvements are still needed in measurement accuracy, sensitivity, and affordability. Several methods that make use of plasmas for atomic emission spectroscopy (AES) of trace metals are being developed to address this need for real time monitoring of heavy metals in smoke stacks. Some of these methods such as inductively coupled plasma (ICP) $)^{2,3}$ and laser induced breakdown spark (LIBS) systems ${ }^{4,5}$ have some limitations. In the case of ICPs they either require high power (more than $2 \mathrm{~kW}$ ) and/or special gases such as argon or helium. In the case of LIBS they analyze a very small volume of the stack gas (few cubic micrometers) in situ for very short time pulses (a few microseconds) which limits sensitivity and may not be representative of the bulk gas. These methods have also not been able to consistently achieve EPA's goal of 20\% relative accuracy to EPA's current reference method using collected stack samples of metals content.

At the Plasma Science and Fusion Center at MIT, a microwave sustained plasma is being developed for real time atomic emission spectroscopy of trace metals in stack exhaust ${ }^{6}$. Unlike other microwave plasma element analysis systems that make use of a surfatron ${ }^{7}$, a resonant cavity ${ }^{8}$ or an Okamoto cavity ${ }^{9}$ to sustain a plasma, the MIT system uses a nonresonant shorted waveguide. This allows operation of a very stable and efficient plasma in air and undiluted stack exhaust at moderate powerlevels $(0.5-1.5 \mathrm{~kW})$ and at high flow rates (>12 l/min). Excellent minimum detection limits have been achieved in air and undiluted stack exhaust plasmas for $\mathrm{Pb}, \mathrm{Be}$, and $\mathrm{Cr}^{10}$. Good detection limits have also been achieved for $\mathrm{Cd}, \mathrm{Hg}$, and $\mathrm{As}$ in pure nitrogen and noble gas plasmas. However, the detection limit for $\mathrm{Hg}$ and $\mathrm{As}$ has been found to degrade significantly in an air plasma.

The purpose of the present study is to attempt an understanding of why the mercury emission light at $253.65 \mathrm{~nm}$ is significantly decreased in a microwave sustained air plasma at atmospheric pressure. The present study has focused on the role of oxygen in the plasma. In the case of arsenic detection at UV wavelengths $<200 \mathrm{~nm}$, oxygen absorption of the UV light can explain the reduced light emission. However, plasma absorption at $253.65 \mathrm{~nm}$ has been ruled out for the case of mercury by the measurements presented here. Other researchers have also found that the addition of some compounds containing oxygen such as carbon dioxide and water vapor to an argon-nitrogen plasma decreases the mercury light intensity to a considerable extent ${ }^{11}$. Also, J.F. Camuna-Aguilar et al. ${ }^{12}$ describe the effect of water on the mercury signal in an argon and helium microwave induced plasma. 


\section{INSTRUMENTATION}

A schematic diagram of the equipment used in this experiment is given by figure 1 .

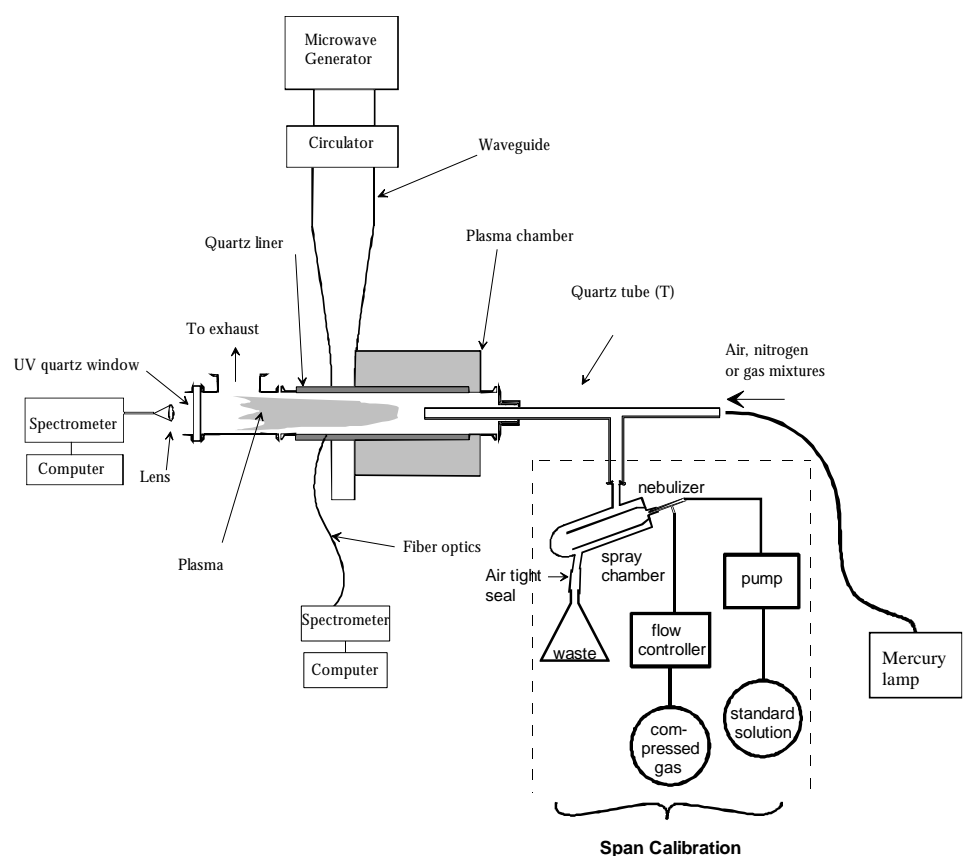

Figure 1. Experimental setup

The microwave source is a $2.45 \mathrm{GHz}$ magnetron from Astex Inc., with a maximum power of $2.5 \mathrm{~kW}$. The microwave radiation travels inside a standard WR-284 brass waveguide which is tapered to inside dimensions of $19.1 \times 76.2 \mathrm{~mm}$ with a short at the tapered end. A $31.8 \mathrm{~mm}$ hole traverses the wide waveguide walls a quarter wavelength back from the short. The plasma discharge is started by a $15 \mathrm{kV}$ electric spark between two tungsten electrodes located just up stream of the waveguide inside the plasma chamber and connected to a high voltage transformer. The plasma is then sustained by the microwave radiation and maintained inside a $25.4 \mathrm{~mm}$ id boron nitride tube in the chamber. It is kept from the walls by a swirl jet of air or nitrogen.

A suction pump draws a gas sample through a $6 \mathrm{~mm}$ id quartz Tee which is inserted into the plasma chamber. After passing through the plasma, the gas is cooled down in a heat exchanger before it goes into the suction pump, then out to the exhaust hood. Metallic aerosols such as mercury are injected into the plasma chamber through the quartz Tee by a Meinhart pneumatic nebulizer with an injection rate of $1 \mathrm{ml} / \mathrm{min}$ and an efficiency of $\sim 0.9 \%$. A $10,000 \mu \mathrm{g} / \mathrm{ml} \mathrm{ICP}$ standard $\mathrm{Hg}$ solution in $5 \% \mathrm{HNO}_{3}$ is used as the source of mercury. For iron and lead, a $1000 \mu \mathrm{g} / \mathrm{ml}$ solution is used. The nebulizer is calibrated against a "rod-insertion calibration method" developed in the laboratory ${ }^{13}$. On the plasma axis and at a distance of $25 \mathrm{~cm}$ from the waveguide, there is a quartz window and a lens that takes the light from the plasma and concentrates it outside at the lens focal length (75 $\mathrm{mm})$. A one or two meter long UV fiber optic is mounted at the focal point and is connected to an Instruments S.A. Model THR-640 spectrometer. This instrument has a 2400 groove/mm grating, adjustable slit, and a Princeton Instruments Model IRY-512W 
intensified 512-element detector array. The spectral resolution is about $0.05 \mathrm{~nm}$ with an instantaneous spectral range of about $6.5 \mathrm{~nm}$ that can be tuned from 180 to $600 \mathrm{~nm}$. When nitrogen spectrum is studied, a custom spectrometer with higher resolution is used to distinguish between the $\mathrm{P}$ and $\mathrm{R}$ branch lines. This instrument has a 3600 groove $/ \mathrm{mm}$ grating, $0.84 \mathrm{~m}$ focal length, and a 2,048 element StellarNet CCD detector array. It is capable of a maximum spectral resolution of about $0.012 \mathrm{~nm}$ centered at $386 \mathrm{~nm}$ in one of its four instantaneous bands used for the present measurements ${ }^{14}$.

Possible ultraviolet light absorption in the plasma at $253.65 \mathrm{~nm}$ was checked by transmitting light from a low-pressure mercury lamp axially through the length of the plasma as shown in Figure 1. The mercury lamp was a Model 6035 from Oriel Corp. The light from this lamp was directed by a fused silica fiber to the axial input of the Tee. For the UV transmission measurements the span calibration system was removed to allow the introduction of the axial flow gas into the plasma through the side port of the Tee. The air, nitrogen, and oxygen gas flows were independently controlled by calibrated flow meters at the input.

\section{EXPERIMENTS AND RESULTS:}

For all experiments, an aerosol of clean deionized water vapor is first injected into the plasma trough the nebulizer to acquire a background spectrum. An aerosol of the solution containing mercury is then introduced into the plasma. The water spectrum is used to subtract the effect of moisture on the mercury spectrum. It is assumed that the water spectrum is representative of the diluted $\mathrm{HNO}_{3}$ acid spectrum. The base plasma gas flows are 14 liter per minute axial and 9 1/min swirl with pure nitrogen in both flows. Oxygen is then added into the axial flow through a very sensitive Omega Model 234 flowmeter. The ISA spectrometer is used to sequentially take 400 instantaneous spectrums at 100 $\mathrm{ms} / \mathrm{spectrum}$ at each oxygen concentration. The data analysis consists of averaging the 400 spectrums for the background and for the signal with mercury injected into the plasma, then subtracting the averaged background signal from the averaged mercury signal. The minimum detection limit (MDL) is calculated as 3 times the standard deviation of the noise of the resulting signal and is given by the following formula:

$$
M D L=\varepsilon \frac{3 \sigma X R}{S F}
$$

where $\varepsilon$ is the nebulizer efficiency, $\sigma$ is the standard deviation of the noise taken on a background detector pixel as close as possible to the spectral signal peak, $\mathrm{X}$ is the standard solution concentration in $\mu \mathrm{g} / \mathrm{ml}, \mathrm{R}$ is the rate of solution in $\mathrm{ml} / \mathrm{min}$ pumped into the nebulizer, $\mathrm{S}$ is the signal intensity in counts, and $\mathrm{F}$ is the volume flow rate of gas in the sample line in $\mathrm{m}^{3} / \mathrm{min}$.

Figure 2 shows the effect of adding oxygen to the nitrogen axial flow on the $253.65 \mathrm{~nm}$ mercury line emission intensity and its minimum detection limit. Mercury signal decreases and the detection limit increases as the oxygen content is increased in the axial flow gas. This effect has not been observed with lead and iron. In fact, the signal of lead on the 
$405.78 \mathrm{~nm}$ line increases slightly with oxygen in the axial flow and the detection limit decreases as shown in Figure3.

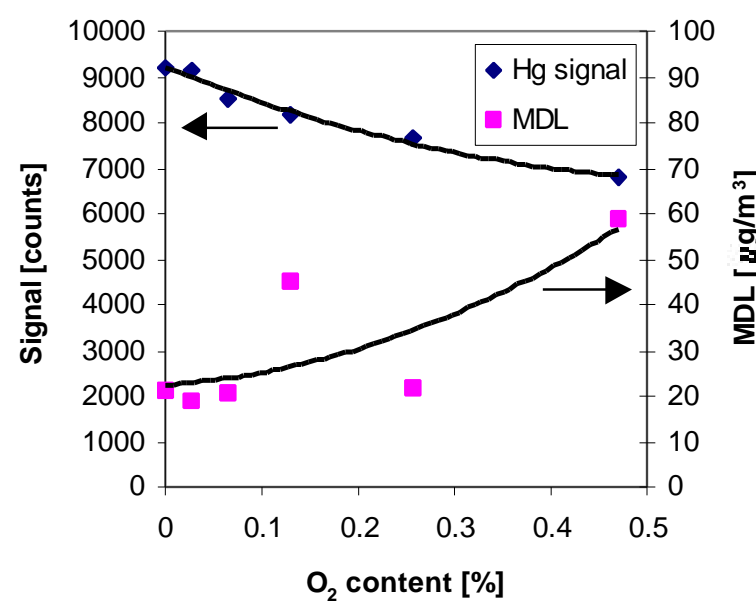

Figure 2. Effect of $\mathrm{O}_{2}$ on $\mathrm{Hg} 253.65 \mathrm{~nm}$ signal and minimum detection limit

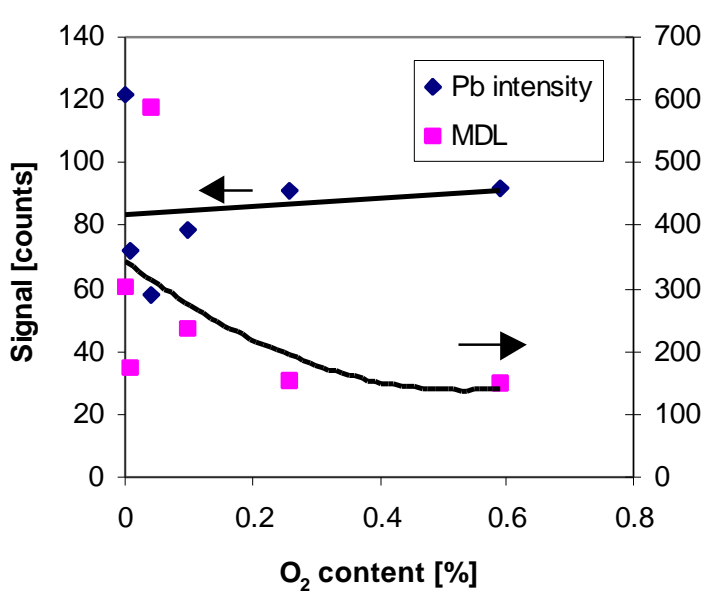

Figure 3. Effect of $\mathrm{O}_{2}$ on $\mathrm{Pb}$ signal and minimum detection limit

From Figure 2, it appears that oxygen and/or an oxygen derived species such as ozone may be responsible for the decrease of $\mathrm{Hg}$ light emission in the nitrogen plasma. To understand this phenomena, we investigated the effect of oxygen on the rotational and the excitation temperatures. The excitation temperature is considered to be representative of the electron temperature.

\subsection{Rotational temperature measurements}

We measured the rotational temperature of the $\mathrm{N}_{2}{ }^{+}$ion in the plasma by using the line pair intensity ratio method ${ }^{15}$. This method assumes a Maxwell-Boltzmann distribution of energy levels and takes the ratio of the light emission intensity between two spectral peaks to obtain a temperature. In our case one peak is taken from each of the $\mathrm{R}$ and $\mathrm{P}$ branches of the nitrogen ion molecule $\mathrm{N}_{2}{ }^{+}$. The line intensity for one peak is given by reference ${ }^{16}$ :

$$
I_{e m}=\frac{C_{e m} \cdot n^{4}}{Q r} \cdot\left(J^{\prime}+J^{\prime \prime}+1\right) \exp \left[-B^{\prime} J^{\prime}\left(J^{\prime}+1\right) h c / k T\right]
$$

where $\mathrm{I}_{\mathrm{em}}$ is the emission intensity of a given line, $\mathrm{C}_{\mathrm{em}}$ is a constant depending on the change of dipole moment and the total number of molecules in the initial vibrational level, $\mathrm{n}$ is the wavelength, J' the upper level, J" the lower level, B' is the rotational constant, and $\mathrm{Q}_{\mathrm{r}}$ is the rotational partition function. By applying equation 2 to R29 and P56 peaks of the $0-0$ band of the first negative system of $\mathrm{N}_{2}{ }^{+}$and by taking the ratio of the two lines, we obtain the rotational temperature through the following equation: 


$$
T_{\text {rot }}[K]=\frac{6421}{\ln \frac{I_{\text {R29 }}}{I_{\mathrm{P} 56}}+0.62415}
$$

These two lines were chosen because they almost overlap spectrally, but their energy levels are far apart. This minimizes the error in the temperature calculation due to spectral variation in the spectrometer response and due to experimental intensity measurement uncertainties.

For these rotational temperature measurements, we used the custom spectrometer described above. Figure 4 shows a partial spectrum of the $\mathrm{N}_{2}{ }^{+}$first negative system 0-0 band and the two peaks, R29 and P56, used to calculate the rotational temperature. Equation 3 was applied to different spectra taken as a function of oxygen concentration in the plasma. The resulting rotational temperature is shown in Figure 5. A rotational temperature of $5000 \mathrm{~K}$ has been determined in an all nitrogen discharge, which is in agreement with the results of K. Ogura et al. ${ }^{17}$ who measured the rotational temperature in a microwave-induced nitrogen plasma using an Okamoto cavity. From this figure, we can see that there is a slight drop in the rotational temperature when oxygen concentration increases in the plasma.

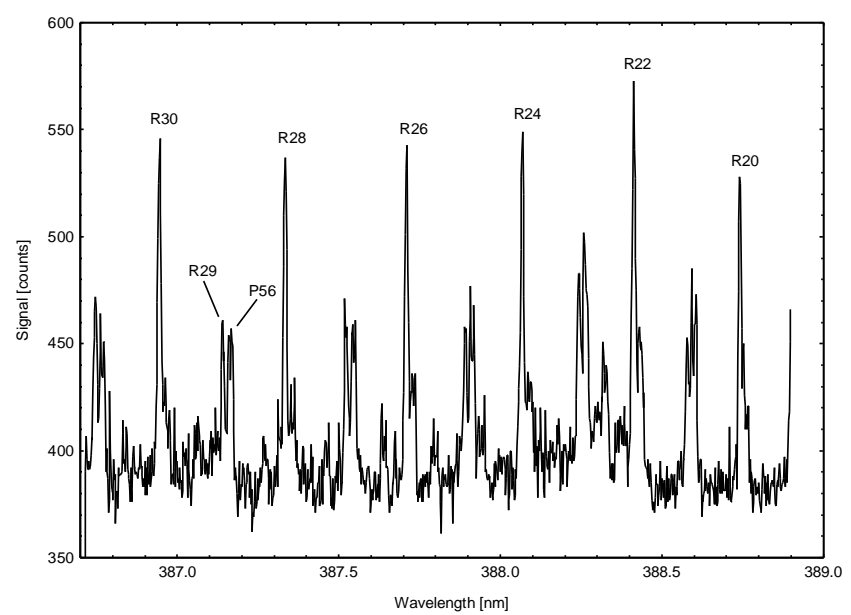

Figure $4 . \mathrm{N}_{2}{ }^{+}$spectrum 


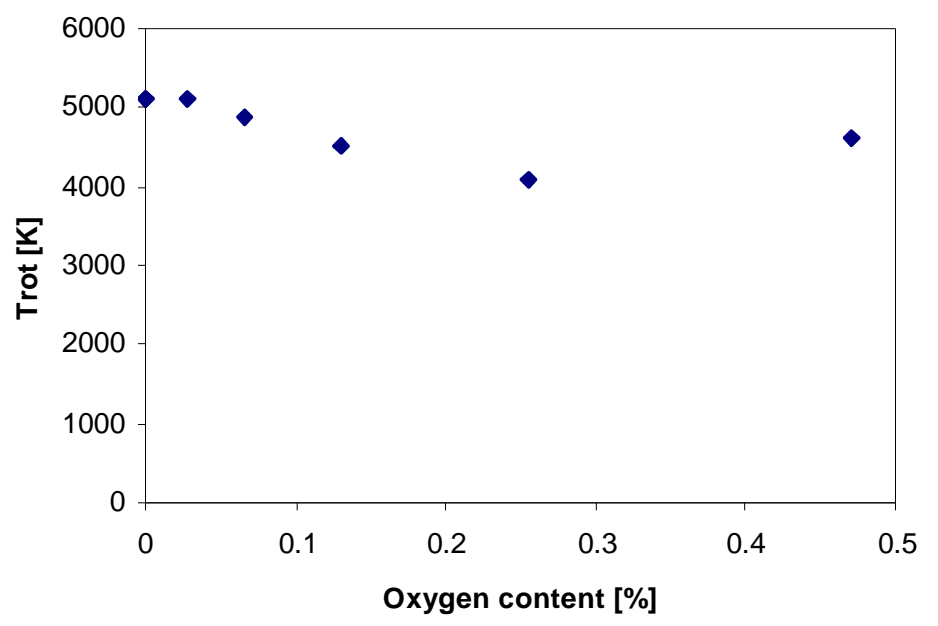

Figure 5. Rotational temperaturee

3.2. Excitation temperature measurements:

The atomic excitation temperature has also been calculated assuming a MaxwellBoltzmann distribution of the electronic energy levels. The slope of a log plot of emission intensity versus the energy of the excited level as related below gives the temperature ${ }^{15}$.

$$
\log \left(\frac{I \lambda}{g f}\right) \alpha \mathrm{E}_{\text {exc }}
$$

where $\mathrm{I}$ is the emission signal intensity, $\lambda$ is the wavelength of the transition, $\mathrm{g}$ is the statistical weight, $f$ the oscillator strength and $E_{e x c}$ is the excitation energy. The slope is related to theexcitation temperature by the following equation:

$$
\mathrm{T}_{\text {exc }}=\frac{-0.625}{\log \frac{n^{3}}{g f}} \bullet E_{\text {exc }}
$$

Because iron lines between 370 and $380 \mathrm{~nm}$ were too weak to use for temperature measurements in a pure nitrogen plasma, we used titanium lines covering an energy range from 30,837 to $43,781 \mathrm{~cm}^{-1}$ to measure the excitation temperature. The values of gf parameters are taken from Wiese and Furt ${ }^{18}$. Figure 6 shows the results as oxygen concentration is increased in a nitrogen plasma. The excitation temperature is within the limits of the results of K. Ogura et al. ${ }^{17}$. Even though the error margin is high, a slight decrease in the excitation temperature can be noticed on the curve. 


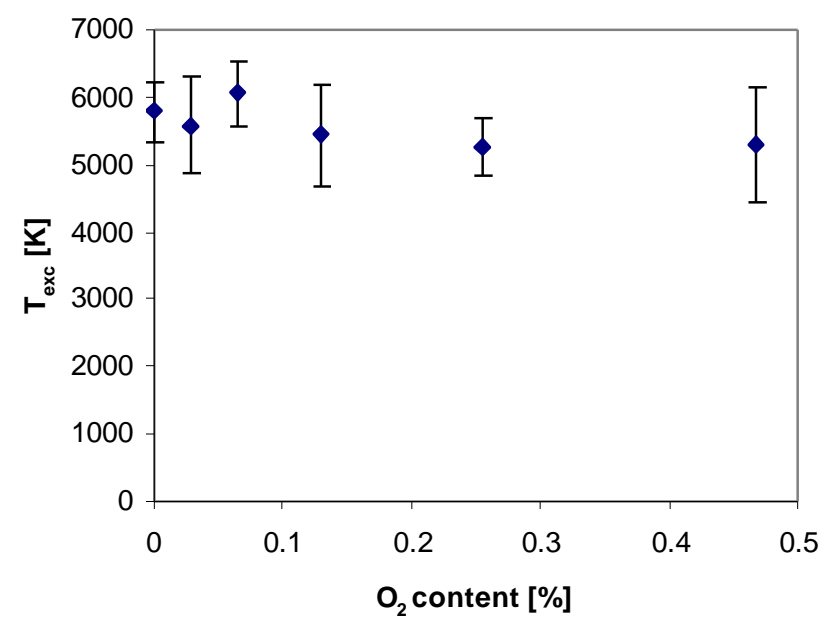

Figure 6. Excitation temperature

\subsection{Absorption}

Transmission measurements of $253.65 \mathrm{~nm}$ light from a low-pressure mercury lamp through the entire axial length of the plasma $(\sim 25 \mathrm{~cm})$ as a function of oxygen concentration in nitrogen did not reveal any measurable increase in absorption at this wavelength. A slight increase in transmission was noted with a small addition $(\sim 2 \%)$ of oxygen, but at $20 \%$ concentration there was no difference, within experimental measurement error, in the UV transmission relative to a pure nitrogen plasma. The slight increase in transmission is probably due to a lensing effect along the plasma axis of propagation. This measurement shows absorption of the $253.65 \mathrm{~nm}$ mercury light by oxygen and/or oxygen derived compounds such as ozone is not the mechanism responsible for the decrease in mercury emission intensity when oxygen is added to the plasma.

\section{DISCUSSION:}

The results presented above clearly show that the addition of oxygen to a nitrogen plasma has an opposite effect on the light emission intensity of mercury at $253.65 \mathrm{~nm}$ and on lead at $405.78 \mathrm{~nm}$ as well as on other transitions of iron. Any explanation of the plasma mechanism that reduces the light emission of mercury by the addition of oxygen should not be in conflict with the fact that oxygen increases the light emission intensity of lead and iron transitions. The present experimental measurements restrict the possible explanations for the observed behavior of mercury emission in an atmospheric microwave plasma.

Selective absorption of the $253.65 \mathrm{~nm}$ mercury light by an oxygen species is ruled out by the UV transmission measurements. The fact that the measured rotational temperature, which is usually assumed to be the same as the gas temperature, is about $5000 \mathrm{~K}$ indicates that most of the oxygen molecules can be decomposed. This would allow the formation of oxygen radicals, therefore producing ozone molecules in the after glow. It is well known that ozone strongly absorbs the 253.65 mercury line. However, the observed lack of absorption indicates that ozone is not present in the regions of the plasma and afterglow 
through which the spectrometer views emission. Since ozone decomposes at a temperature of $261.1^{\circ} \mathrm{C}^{19}$, this suggests that the plasma and adjacent regions being viewed are too hot for the formation of ozone.

Not tested by the present absorption measurement is the effect of trace mercury in the plasma. Absorption by unexcited mercury in the outer regions of the plasma chamber through which the plasma emission is viewed may be a factor in reducing observed mercury emission since the lower level of the $253.65 \mathrm{~nm}$ transition is the ground state. This by itself would not explain the observed change between a pure nitrogen and nitrogen with oxygen plasma having the same content of mercury. The explanation of the observed decrease in mercury emission with the addition of oxygen is most likely due to a reduction in the excitation of mercury when oxygen is present in the plasma.

Oxygen, having an affinity for electrons, may be expected to reduce the electron density in the plasma, therefore reducing the probability for a mercury atom to be excited by electron impact either directly or via a metastable state of another plasma species. This explanation is unlikely because electron impact, either directly or indirectly, is responsible for exciting all atomic species in the plasma. A reduction in electron density by itself would be expected to effect all atomic emission in the same way. This is not the case in the present observations. Lead and other metal emission line intensities increase opposite to the oxygen effect on mercury.

A change in the electron energy distribution or temperature with oxygen could account for the observed behavior of the metals emission. The electron impact cross-section for the excitation of the various mercury transitions as a function of electron energy is shown in Figure $7^{20}$. The excitation curve for the strongest $253.65 \mathrm{~nm}$ transition is given along with the excitation curves for the $435.8,407.8,313.2,313.2,312.6$, and $289.4 \mathrm{~nm}$ lines and their sum with the $265.4 \mathrm{~nm}$ line. The excitation cross-section for the $253.65 \mathrm{~nm}$ transition peaks at a very narrow electron energy bandwidth at $5.5 \mathrm{eV}$. This is much higher than the measured plasma excitation temperature of approximately $0.5 \mathrm{eV}$, which is considered an indicator of the mean electron energy. It is the energetic tail electrons of the electron energy distribution that excite mercury in this plasma. At the maximum cross section for the line at $253.65 \mathrm{~nm}$, a decrease in the electron energy of $12.5 \%$ would cause a 50\% decrease in the light intensity. Also, a slight decrease observed for the excitation temperature as shown in Figure 3 could indicate a larger reduction in energetic electrons. This may account for the observed decrease in signal intensity of the mercury line when oxygen is added to the nitrogen plasma. The other metals behave differently because their excitation cross-section may not be as peaked and shifted to lower energies. 


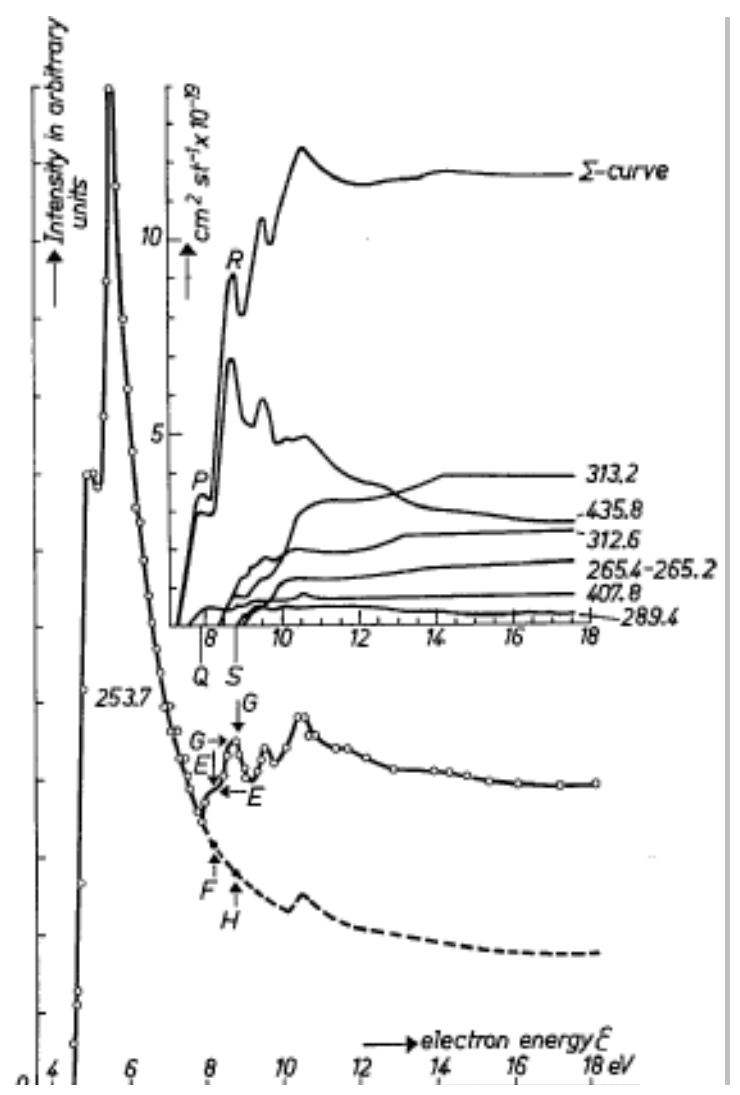

Figure 7. Electron impact cross-section for the excitation of the various mercury transitions as a function of electron energy (from ref. 20)

Another possible explanation for the observed behaviour of metals light emission is the selective quenching of excited mercury atoms by oxygen or oxygen derived species in the plasma. This possibility is a subject for future investigations.

\section{CONCLUSION}

This study has shown that oxygen addition to a nitrogen atmospheric plasma effects the light emission from mercury and lead in opposite ways. Mercury light emission of the $253.65 \mathrm{~nm}$ line is strongly reduced by the presence of oxygen in the plasma while the lead $405.78 \mathrm{~nm}$ line and some other metal lines are enhanced. Selective absorption of the mercury UV emission by oxygen or oxygen derived species in the plasma has been rule out by UV transmission measurements. It has been shown that oxygen slightly lowers the gas temperature and the excitation energy in the plasma. This effect in combination with the sharp excitation cross-section of mercury may account for the decrease in the light emission intensity of mercury with the addition of oxygen. An improved understanding of the plasma mechanisms that effect the emission intensity of mercury in atmospheric plasmas is of value to the development of real time mercury trace metals pollution monitors. 


\section{REFERENCES}

1 G. A. Meyer, Proceedings of SPIE, Vol. 3534, Environmental Monitoring and Remediation Technologies, Boston, Nov. 1998.

2 C. Trassy, R. Diemiaszonek, P. Pasquini, and R. Meunier: Proc. Of Int. Sym. on Environmental Technologies, Plasma System and Applications, Atlanta, 1995, p. 401.

3 M. D. Seltzer and R. B. Green: Process Control and Quality. 6 (1994) No. 1, 37.

4 D. W. Hahn: Appl. Phys. Lett. 72 (1998), 2960.

5 J. P. Singh, Fang-Yu Yueh, Hansheng Zhang, R.L. Cook: Process Control and Quanlity 10 (1997), 247.

6 P.P. Woskov, D.Y. Rhee, P. Thomas, D.R. Cohn, J.E. Surma, and C. Titus: Rev. Sci. Instrum. 67 (1996), 3700.

7 M.H. Abdallah, S. Coulombe, and J.M. Mermet: Spectrochemica Acta 37B (1982), No. $7,583$.

8 K. Larjava, T. Laitinen, T. Vahlman, S. Artmann, V. Siemens, J.A.C. Broekaert, and D. Klockow: Intern. J. Environ. Anal. Chem. 49 (1992), 73.

9 Y. Okamoto: Anal. Sci. 7 (1991), 283.

10 P.P. Woskov, K. Hadidi, P. Thomas, K. Green, G. Flores, and D. Lamar: Massachusetts Institute of Technology, PSFC/RR-98-1, (1998).

11 V. Siemens, T. Harju, T. Laitinen, K. Larjava, and J.A.C. Broekaert: Fresenius J. Anal. Chem 351 (1995), 11.

12 J.F. Camuna-Aguilar, R. Pereiro-Garcia, J.E. Sanchez-Uria, and A. Sanz-Medel: Spectrochemica Acta 49B N0.5, (1994), 475.

13 G.J. Flores, K.M. Green, P.P. Woskov, K. Hadidi, and P. Thomas: 40th Annual Meeting of the Division of Plasma Physics of APS, Nov. 1998, New Orleans, LA.

14 P. P. Woskov, K. Hadidi, M. C. Borras, P. Thomas, K. Green, G. J. Flores, Rev. Sci. Instrum 70 Jan. 1999, to be published

15 P.W.J.M. Boumans: Indusctively coupled plasma, Emission spectroscopy, part 2, eds. Wiley interscience, 1987, chapter 10, 353.

16 G. Herzberg: Molecular spectra and molecular structure, spectra of diatomic molecules, part 1, eds. Van Nostrand Reinhold Company, 1950, chapter 3, 126. 
17 K. Ogura, H. Yamada, Y. Sato, and Y. Okamoto: Applied Spectroscopy 51 No. 10, (1997), 1496.

18 W.L. Wiese and J.R. Furh: J. Chem. Ref. Data 4 (1975), 263.

19 CRC Handbook of Chemistry and Physics. David R. Lide Editor in Chief, 76th edition, 1995-1996.

$20 \mathrm{~J}$. Hermanus Maria: Measurements of optical excitation function of the mercury atom, Proefschrift-Utrecht publisher, 1961. 